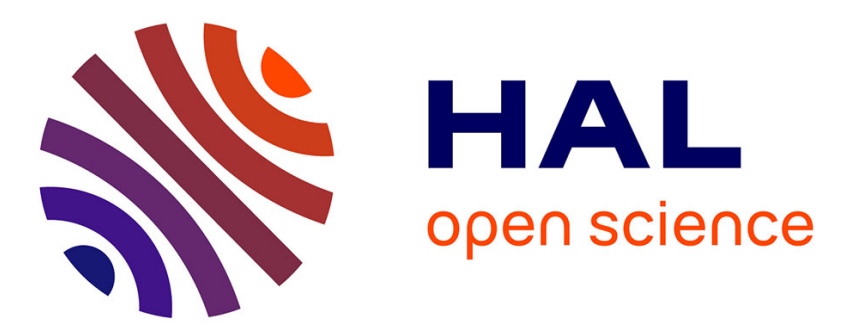

\title{
Coupling BEM with FEM by a direct computation of the boundary stiffness matrix
}

Guy Bonnet, Abdelghani Seghir, Alain Corfdir

\section{To cite this version:}

Guy Bonnet, Abdelghani Seghir, Alain Corfdir. Coupling BEM with FEM by a direct computation of the boundary stiffness matrix. Computer Methods in Applied Mechanics and Engineering, 2009, 198 (30-32), pp.2439-2445. 10.1016/j.cma.2009.02.032 . hal-00691016

\section{HAL Id: hal-00691016 https://hal.science/hal-00691016}

Submitted on 17 Jan 2016

HAL is a multi-disciplinary open access archive for the deposit and dissemination of scientific research documents, whether they are published or not. The documents may come from teaching and research institutions in France or abroad, or from public or private research centers.
L'archive ouverte pluridisciplinaire HAL, est destinée au dépôt et à la diffusion de documents scientifiques de niveau recherche, publiés ou non, émanant des établissements d'enseignement et de recherche français ou étrangers, des laboratoires publics ou privés. 


\title{
Coupling BEM with FEM by a direct computation of the boundary stiffness matrix
}

\author{
G. Bonnet ${ }^{\mathrm{a}, *}$, A. Seghir ${ }^{\mathrm{a}, \mathrm{c}}, \mathrm{A}$. Corfdir $^{\mathrm{b}}$ \\ a Université Paris-Est, Laboratoire de Modélisation et Simulation Multi-Echelle (FRE CNRS 3160), Institut Navier, 5 Boulevard Descartes, 77454 Marne la Vallée Cedex, France \\ ${ }^{\mathrm{b}}$ Université Paris-Est, UMR Navier-CERMES, Institut Navier, Ecole Nationale des Ponts et Chaussées, 6-8 avenue Blaise Pascal, 77455 Marne la Vallée Cedex 2, France \\ ' Université A.Mira de Bejaia, Département de Génie Civil, 06000 route de Targua Ouzemour, Bejaia, Algeria
}

\section{A B S T R A C T}

The "boundary stiffness matrix" characterizing in a discretized form the response of the boundary to given Dirichlet boundary conditions allows the coupling between BEM and FEM. The paper presents an approach based on the direct computation of the stiffness matrix from the potential function related to a given Dirichlet boundary condition. The method produces a symmetric stiffness matrix as for the Singular Galerkin boundary element method, but does not need to compute hypersingular integrals. In addition, the method uses only the nodal values of the boundary potential, but does not need discretized values of the normal gradient at the boundary, as for usual boundary element methods, which reduces the number of matrices to be constructed. The regularization of the integrals is achieved by means used for (simply) singular integrals introduced for the collocation boundary element method. An example of application is produced in the case of the solution of Laplace equation within a plane domain.

Keywords:

Boundary element

Symmetric

Stiffness matrix

Finite element

Regularization

\section{Introduction}

The approximate solution of boundary value problems using boundary integral equation and boundary elements uses very often the popular method of collocation. It is well known that such a method leads to non-symmetrical linear discretized systems. Such a situation is unsatisfactory for the following reasons: First, when coupling with finite elements, it is necessary to use a method of solution of the discretized linear system of equations which has no symmetry properties. The main unsatisfactory consequence is however that the stiffness matrix deduced from the linear system being unsymmetrical, it is no more possible to comply to the reciprocity theorem, both properties being closely related $[1,6,7,17,21]$. This situation led to the formulation of the "Symmetric Galerkin Boundary Element Method" $[4,6,22,24,25,27,28]$. This method induces numerical difficulties when applications are in view, because the kernels involved in the integrals produced by this formulation are strongly singular, using second derivatives of the Green's function, so that, even now, results are produced by using the usual collocation method [2]. The same can be said of the method proposed by $[10,20]$, where strongly singular kernels appear. From another point of view, the non-singular methods using sources outside the domain $[12,31]$ lead to other kinds of numerical problems.

Even if it is possible to reduce the singularity by a convenient regularization $[4,5]$, or by using closed-form expressions $[3,8,23,30]$,

\footnotetext{
* Corresponding author.

E-mail address: guy.bonnet@univ-paris-est.fr (G. Bonnet).
}

the presence of a hypersingular kernel remains a serious difficulty and led for example to imagine the use of Fourier transforms to compute the matrix elements [11].

Taking into account the numerical efficiency of the finite element method, it seems that the best field of application of boundary element methods is to modelize infinite domains containing a material characterized by linear properties coupled with a finite domain. For example, recent works were produced to achieve this objective $[16,29]$ by specific methods to perform this kind of coupling without matrix inversion. In this context, it seems that a main objective is to build the boundary stiffness matrix characterizing the boundary response to a Dirichlet boundary condition applied to the boundary of the infinite domain. This is the point of view adopted when using the "Dirichlet to Neumann Finite Element Method" (DtN FEM) [13-15,18,19]. A "Dirichlet to Neumann operator" built from the first Green's function of the infinite domain produces directly the boundary stress field induced within the infinite domain by a given Dirichlet boundary condition. The condensation of that operator on the nodes of the boundary produces directly the stiffness matrix, without matrix inversion, which is a main advantage when boundary discretization points on the boundary are numerous. However, the main difficulty is that the DtN-FEM needs the first Green's function, which is known only for some specific types of boundary shapes and operators, while other boundary element formulations need only the Green's function for the overall space and can be used for any shape of the boundary.

The purpose of the paper is to present a method allowing to build a symmetric boundary element formulation leading to a 
discretized symmetrical boundary stiffness matrix. The method is intermediate between the classical "Singular Galerkin Boundary Element Method" and the "Dirichlet to Neumann Finite Element Method". The proposed method uses the classical fundamental solution of the operator (Green's function for the infinite domain) as for the SGBEM, but, instead of using a Galerkin approach, the normal gradient at the boundary induced by a given boundary field along the boundary (Dirichlet boundary condition) is estimated by using an indirect method. It allows to estimate a potential function induced by a given Dirichlet condition, from which the stiffness matrix can be obtained. The method is presented in the case of Laplace operator.

\section{Computation of the potential function related to a given Dirichlet boundary condition}

Let us consider the solution $u$ of the Laplace equation within a domain $D$, related to a given Dirichlet boundary condition $u=\bar{u}$, where $\bar{u}$ is known. Using an indirect approach, the harmonic function $u$ inside $D$ is sought by using a repartition of sources located along the boundary by:

$u(\mathbf{y})=\int_{\partial D} G(\mathbf{z}, \mathbf{y}) \Phi(\mathbf{z}) \mathrm{d} S(\mathbf{z})$.

Taking the derivative of this relation allows the obtaining of:

$q(\mathbf{y})=\frac{\partial u(\mathbf{y})}{\partial \mathbf{n}(\mathbf{y})}=\int_{\partial D} \frac{\partial G(\mathbf{x}, \mathbf{y})}{\partial \mathbf{n}(\mathbf{y})} \Phi(\mathbf{x}) \mathrm{d} S(\mathbf{x})$.

From this, it is possible to define a potential function equal to the supply of energy $W$ when the boundary condition increases smoothly from 0 to $\bar{u}$, given by:

$W=\frac{1}{2} \int_{\hat{a} D} q(\mathbf{y}) u(\mathbf{y}) \mathrm{d} S(\mathbf{y})$.

Finally, $W$ is given by:

$W=\frac{1}{2} \int_{\partial D}\left[\int_{\partial D} \frac{\partial G(\mathbf{x}, \mathbf{y})}{\partial \mathbf{n}(\mathbf{y})} \Phi(\mathbf{x}) \mathrm{d} S(\mathbf{x}) \int_{\partial D} G(\mathbf{z}, \mathbf{y}) \Phi(\mathbf{z}) \mathrm{d} S(\mathbf{z})\right] \mathrm{d} S(\mathbf{y})$.

It is easily seen that the formulation involves a singular integral, due to the term $\partial G(\mathbf{x}, \mathbf{y}) / \partial \mathbf{n}(\mathbf{y})$. It is however possible to get a regularized formulation along the lines of $[6,26]$ introduced for the classical collocation boundary element method, as described below. The interior and exterior problems are successively treated.

\subsection{Regularization for the interior problem}

Let us consider that the sources $\Phi(\mathbf{x})$ are continuous and defined not only on the boundary $\partial D$ of $D$, but also within its neighbourhood. For the interior problem, the integral in (4) may be considered as the limit of a similar expression $W_{1}$, the sources being external to the domain. As a consequence, let us consider that the sources are located on the boundary of a domain $D_{0}$ containing $D$.

Eq. (4) becomes:

$W_{1}=\frac{1}{2} \int_{\partial D}\left[\int_{\partial D_{0}} \frac{\partial G(\mathbf{x}, \mathbf{y})}{\partial \mathbf{n}(\mathbf{y})} \Phi(\mathbf{x}) \mathrm{d} S(\mathbf{x}) \int_{\partial D_{0}} G(\mathbf{z}, \mathbf{y}) \Phi(\mathbf{z}) \mathrm{d} S(\mathbf{z})\right] \mathrm{d} S(\mathbf{y})$.

Let us denote by $u_{0}$ the field induced by the repartition of sources along $\partial D_{0}$. Eq. (5) becomes:

$W_{1}=\frac{1}{2} \int_{\partial D}\left[\int_{\partial D_{0}} \frac{\partial G(\mathbf{x}, \mathbf{y})}{\partial \mathbf{n}(\mathbf{y})} \Phi(\mathbf{x}) \mathrm{d} S(\mathbf{x}) \cdot u_{0}(\mathbf{y})\right] \mathrm{d} S(\mathbf{y})$.

Exchanging the order of integration between $\partial D$ and $\partial D_{0}$ leads
$W_{1}=\frac{1}{2} \int_{\partial D_{0}} \Phi(\mathbf{x})\left[\int_{\partial D} \frac{\partial G(\mathbf{x}, \mathbf{y})}{\partial \mathbf{n}(\mathbf{y})} \cdot u_{0}(\mathbf{y}) \mathrm{d} S(\mathbf{y})\right] \mathrm{d} S(\mathbf{x})$.

The limit of that expression when $\partial D_{0}$ tends to $\partial D$ is now sought along the following lines. The Green's function $G$ can be integrated even if the source is on the integration contour. The limit of (7) when $\partial D_{0}$ tends to $\partial D$ is therefore the limit of:

$W_{2}=\frac{1}{2} \int_{\hat{\partial} D_{0}} \Phi(\mathbf{x})\left[\int_{\partial D} \frac{\partial G(\mathbf{x}, \mathbf{y})}{\partial \mathbf{n}(\mathbf{y})} \cdot u(\mathbf{y}) \mathrm{d} S(\mathbf{y})\right] \mathrm{d} S(\mathbf{x})$.

Let us now consider the integral on $\partial D$, denoted by $I$, where $\partial G(\mathbf{x}, \mathbf{y}) / \partial \mathbf{n}(\mathbf{y})$ is denoted by $H(\mathbf{x}, \mathbf{y})$

$I=\int_{\partial D} H(\mathbf{x}, \mathbf{y}) \cdot u(\mathbf{y}) \mathrm{d} S(\mathbf{y})$.

That integral is singular when $\mathbf{x}$ is on $\partial D$. It can be regularized as in $[6,26]$, if the function $u$ presents sufficient regularity conditions. The regularization proceeds as follows: The point $\mathbf{x}$ is external to $D$ and therefore:

$\int_{a D} H(\mathbf{x}, \mathbf{y}) \mathrm{d} S(\mathbf{y})=0$.

Substracting that expression from (9) after multiplication by $u(\mathbf{x})$ leads to:

$I=\int_{\partial D} H(\mathbf{x}, \mathbf{y})[u(\mathbf{y})-u(\mathbf{x})] \mathrm{d} S(\mathbf{y})$.

Such an integral is now regular when $\mathbf{x}$ is on $\partial D$. Finally, the potential function $W$ can be obtained from the following expression, using similar arguments as those used by [5]

$W=\lim _{a D_{0} \rightarrow a D} W_{1}=\lim _{\partial D_{0} \rightarrow a D} W_{2}$

$=\frac{1}{2} \int_{\mathrm{A} D} \Phi(\mathbf{x})\left[\int_{\mathrm{A} D} H(\mathbf{x}, \mathbf{y})[u(\mathbf{y})-u(\mathbf{x})] d S(\mathbf{y})\right] d S(\mathbf{x})$.

Where the difference $u(\mathbf{y})-u(\mathbf{x})$ is given from Eq. (1) by:

$u(\mathbf{y})-u(\mathbf{x})=\int_{\partial D}[G(\mathbf{z}, \mathbf{y})-G(\mathbf{z}, \mathbf{x})] \Phi(\mathbf{z}) \mathrm{d} S(\mathbf{z})$.

\subsection{Regularization for the exterior problem}

In this case, the same considerations can be applied, taking into account that, for the exterior problem, the sources are now within the interior part $D_{i}$ of the domain delimited by the boundary $\partial D$. In addition, the normal to the exterior domain $D$ delimited by $\partial D$ is now directed along the direction which is the opposite to the external normal to $D_{i}$, which allows to replace Eq. (10) by:

$\int_{\partial D} H(\mathbf{x}, \mathbf{y}) \mathrm{d} S(\mathbf{y})-1=0$.

It leads to:

$W=\frac{1}{2} \int_{\partial D} \Phi(\mathbf{x})\left[u(\mathbf{x})+\int_{\partial D} H(\mathbf{x}, \mathbf{y})[u(\mathbf{y})-u(\mathbf{x})] \mathrm{d} S(\mathbf{y})\right] \mathrm{d} S(\mathbf{x})$.

Finally, the expression of the potential function related to a given Dirichlet boundary condition $u(\mathbf{y})$ (in absence of sources existing within $D$ ) is given by Eq. (16) where the sources $\Phi$ are related to the boundary potential by Eq. (1).

\section{Symmetry of the operator appearing in the potential function}

As explained before, one of the main problems when dealing with boundary element formulations is to keep the properties of symmetry which are basically related to the Maxwell-Betti reciprocity theorem. It is straightforward to show that the present 
formulation does not affect the symmetry. The conservation of the symmetry is indeed fundamentally due to the compatibility between the function $u$ and its normal gradient which are computed from the same sources as pointed by $[4,17]$. The symmetry results directly from the symmetry of the operator used to compute the potential function $W$, as shown below.

Let us start from Eq. (5), but let us consider this time the potential function coming from surface sources $\Phi_{1}=\Phi(\mathbf{x})$ and from the surface fluxes induced by the sources $\Phi_{2}=\Phi(\mathbf{z})$. This potential function can be written as:

$W\left(\Phi_{1}, \Phi_{2}\right)=\frac{1}{2} \int_{\mathrm{\partial D}_{0}} \int_{\mathrm{\partial D}_{0}} K(\mathbf{x}, \mathbf{z}) \Phi_{2} \Phi_{1} \mathrm{~d} S(\mathbf{x}) \mathrm{d} S(\mathbf{z})$,

where the kernel appearing in that equation is given by:

$K(\mathbf{x}, \mathbf{z})=\int_{\partial D} \frac{\partial G(\mathbf{x}, \mathbf{y})}{\partial \mathbf{n}(\mathbf{y})} G(\mathbf{z}, \mathbf{y}) \mathrm{d} S(\mathbf{y})$.

Using Green's theorem leads to:

$K(\mathbf{x}, \mathbf{z})=\int_{D} G(\mathbf{z}, \mathbf{y}) \cdot \Delta G(\mathbf{x}, \mathbf{y}) \mathrm{d} V+\int_{D} \frac{\partial G(\mathbf{x}, \mathbf{y})}{\partial \mathbf{y}} \cdot \frac{\partial G(\mathbf{z}, \mathbf{y})}{\partial \mathbf{y}} \mathrm{d} V$,

where $\Delta$ denotes Laplace operator. Point $\mathbf{x}$ being outside the domain $D$, the first integral is equal to zero and finally:

$K(\mathbf{x}, \mathbf{z})=\int_{D} \frac{\partial G(\mathbf{x}, \mathbf{y})}{\partial \mathbf{y}} \cdot \frac{\partial G(\mathbf{z}, \mathbf{y})}{\partial \mathbf{y}} \mathrm{d} V$.

This integral is obviously symmetric with regard to $\mathbf{x}$ and $\mathbf{z}$, and hence:

$K(\mathbf{x}, \mathbf{z})=K(\mathbf{z}, \mathbf{x})$

Introducing this result within Eq. (17) leads to the symmetry of

$W\left(\Phi_{1}, \Phi_{2}\right)=W\left(\Phi_{2}, \Phi_{1}\right)$.

This result is in fact a direct application of the "Maxwell-Betti" theorem of reciprocity in the case of Laplace equation.

\section{Discretization of the potential function and determination of the stiffness matrix}

The aim of this section is to approximate Eqs. (13) and (14) for the interior problem and Eqs. (14)-(16) for the exterior problem in order to obtain a discretized form of the work $W$ as a function of the nodal values of $u$ at boundary nodes. Such an expression of $W$ being a second order polynomial of the nodal values of the harmonic function along the boundary will lead naturally to the stiffness matrix which characterizes the boundary coupling. The method is displayed for the case of plane problems, beginning with the exterior problem.

4.1. Case of the exterior problem

4.1.1. Discretization of the potential function

The discretization of expression (16) leads to the discretized stiffness matrix by using the following linear boundary interpolation functions:

$\mathbf{y}=\sum_{s=1}^{2} N_{s}(\xi) \mathbf{y}_{s}$

and

$\Phi(\mathbf{y})=\sum_{p=1}^{2} N_{p}(\xi) \Phi\left(\mathbf{y}_{p}\right)$,

where $\mathbf{y}_{p}$ denotes discretization points on the surface $\partial D$ and $\xi$ denotes curvilinear coordinates.
In the following, linear boundary elements $e_{m}$ are used. It means that $p$ and $s$ take the values 1 and 2 .

$W=\frac{1}{2} \sum_{m} \int_{e_{m}} \Phi(\mathbf{x})\left[u(\mathbf{x})+\sum_{n} \int_{e_{n}} H(\mathbf{x}, \mathbf{y})[u(\mathbf{y})-u(\mathbf{x})] \mathrm{d} S(y)\right] \mathrm{d} S(\mathbf{x})$

and

$u(\mathbf{y})-u(\mathbf{x})=\sum_{r} \int_{e_{r}}[G(\mathbf{z}, \mathbf{y})-G(\mathbf{z}, \mathbf{x})] \Phi(\mathbf{z}) \mathrm{d} S(\mathbf{z})$.

Relations (25) and (26) show that three integrals must be performed on the boundary, meaning that three different curvilinear coordinates must be used.

Discretization on $e_{m}, e_{n}$ and $e_{r}$ involves the global variable $\mathbf{x}, \mathbf{y}$ and $\mathbf{z}$, and the curvilinear coordinate $\xi, \eta, \zeta$, leading to:

$$
\begin{aligned}
W= & \frac{1}{2} \sum_{m} \int_{e_{m}} \Phi(\mathbf{x}(\xi)) \\
& \times\left[u(\mathbf{x})+\sum_{n} \int_{e_{n}} H(\mathbf{x}, \mathbf{y})[u(\mathbf{y})-u(\mathbf{x})] \mathrm{d} S(\mathbf{y}(\eta))\right] \mathrm{d} S(\mathbf{x}(\xi)),
\end{aligned}
$$$$
u(\mathbf{y})-u(\mathbf{x})=\sum_{r} \int_{e_{r}}[G(\mathbf{z}, \mathbf{y})-G(\mathbf{z}, \mathbf{x})] \Phi(\mathbf{z}(\zeta)) \mathrm{d} S(\mathbf{z}(\zeta)),
$$

where $\Phi(\mathbf{x}(\xi))=N_{1}(\xi) \cdot \Phi\left(\mathbf{x}_{m}\right)+N_{2}(\xi) \cdot \Phi\left(\mathbf{x}_{m+1}\right)$, and a similar expression is taken for $\Phi(\mathbf{z}(\zeta))$.

This sum can be expressed as:

$W=\frac{1}{2} \sum M_{a b} \Phi_{a} \Phi_{b}=\frac{1}{2}[\Phi]^{T}[M][\Phi]$,

where $\Phi_{a}$ and $\Phi_{b}$ are the values of the source $\Phi$ at the different discretization points. $[\Phi]$ is the column vector where the values $\Phi_{b}$ are stored.

The symmetry of the matrix $M$ results directly from the symmetry of $W$. Indeed, introducing two discretized boundary fields $\Phi_{1}$ and $\Phi_{2}$ within the reciprocity property (22) leads to:

$W=\frac{1}{2} \sum M_{a b} \Phi_{1 a} \Phi_{2 b}=\frac{1}{2} \sum M_{a b} \Phi_{2 a} \Phi_{1 b}$

$$
=\frac{1}{2} \sum M_{b a} \Phi_{2 b} \Phi_{1 a},
$$

where the discretized values of the sources $\Phi_{l}$ are denoted by $\Phi_{l a}, \Phi_{l b}$ It leads finally to the symmetry of the matrix $M_{a b}$.

The computation of the matrix elements $M_{a b}$ proceeds as follows: the contributions of all triplets $\left(e_{m}, e_{n}, e_{r}\right)$ to the partial matrix element $M_{a b}$ are given below.

(A) For the free terms, it means the terms corresponding to $u(\mathbf{x})$ alone in (16) the contributions are:

$$
\begin{aligned}
M_{a b} & =M_{1}\left(e_{m}, e_{r}, p, q\right) \\
& =\int_{e_{m}} N_{p}(\xi) J(\xi) d \xi\left[\int_{e_{r}} G(\mathbf{z}(\zeta), \mathbf{x}(\xi)) N_{q}(\zeta) J(\zeta) d \zeta\right]
\end{aligned}
$$

where $J$ is the Jacobian of the transformation induced by the interpolation function: $a=m+p-1, b=r+q-1$, with $p=1,2$ and $q=1,2 . m, n, r$ take the values from 1 to the number of elements N. $a$ and $b$ vary also from 1 to $N$.

In addition, for a closed contour, $a, b$ take the values $a^{\prime}=a-N$ and $b^{\prime}=b-N$ when $a>N$ and $b>N$.

$\xi, \eta, \zeta$ are the local coordinates on the elements $e_{m}, e_{n}, e_{r}$.

$N_{1}(\xi)=(1-\xi) / 2, N_{2}(\xi)=(1+\xi) / 2$.

The contribution of one couple of elements $\left(e_{m}, e_{r}\right)$ can be synthetized within the following matrices

When $e_{m}$ and $e_{r}$ have no common points, the following matrix is obtained, related to the lines and columns $(m, m+1, r, r+1)$ : 


$$
\left(\begin{array}{cccc}
0 & 0 & \frac{1}{2} M_{1}\left(e_{m}, e_{r}, 1,1\right) & \frac{1}{2} M_{1}\left(e_{m}, e_{r}, 1,2\right) \\
0 & 0 & \frac{1}{2} M_{1}\left(e_{m}, e_{r}, 2,1\right) & \frac{1}{2} M_{1}\left(e_{m}, e_{r}, 2,2\right) \\
\frac{1}{2} M_{1}\left(e_{m}, e_{r}, 1,1\right) & \frac{1}{2} M_{1}\left(e_{m}, e_{r}, 2,1\right) & 0 & 0 \\
\frac{1}{2} M_{1}\left(e_{m}, e_{r}, 1,2\right) & \frac{1}{2} M_{1}\left(e_{m}, e_{r}, 2,2\right) & 0 & 0
\end{array}\right) .
$$

When $e_{m}$ and $e_{r}$ have one common point and $r=m+1$ the contribution is a matrix related to lines and columns $(m, m+1, m+2)$ :

$\left(\begin{array}{ccc}0 & \frac{1}{2} M_{1}\left(e_{m}, e_{r}, 1,1\right) & \frac{1}{2} M_{1}\left(e_{m}, e_{r}, 1,2\right) \\ \frac{1}{2} M_{1}\left(e_{m}, e_{r}, 1,1\right) & M_{1}\left(e_{m}, e_{r}, 2,1\right) & \frac{1}{2} M_{1}\left(e_{m}, e_{r}, 2,2\right) \\ \frac{1}{2} M_{1}\left(e_{m}, e_{r}, 1,2\right) & \frac{1}{2} M_{1}\left(e_{m}, e_{r}, 2,2\right) & 0\end{array}\right)$

When $e_{m}$ and $e_{r}$ have one common point and $r=m-1$ the contribution is a matrix related to lines and columns $(m-1, m, m+1)$ :

$\left(\begin{array}{ccc}0 & \frac{1}{2} M_{1}\left(e_{m}, e_{r}, 1,1\right) & \frac{1}{2} M_{1}\left(e_{m}, e_{r}, 2,1\right) \\ \frac{1}{2} M_{1}\left(e_{m}, e_{r}, 1,1\right) & M_{1}\left(e_{m}, e_{r}, 1,2\right) & \frac{1}{2} M_{1}\left(e_{m}, e_{r}, 2,2\right) \\ \frac{1}{2} M_{1}\left(e_{m}, e_{r}, 2,1\right) & \frac{1}{2} M_{1}\left(e_{m}, e_{r}, 2,2\right) & 0\end{array}\right)$.

When $e_{m}$ and $e_{r}$ are identical, the contribution is a matrix related to lines and columns $(m, m+1)$ :
That integral is equal to:

$\left.J=\int_{\zeta=-1}^{\zeta=1} 2 N_{q} \ln (\|\mathbf{V}\|)+N_{q} \cdot \ln \left(\zeta^{2}+2 \cdot \cos (\theta) d \cdot \zeta\right)+d^{2}\right) \mathrm{d} \zeta$,

where $\theta$ is the angle between $\mathbf{x}_{c}$ and $\mathbf{V}$ and $d=\left\|\mathbf{x}_{c}\right\| /\|\mathbf{V}\|$.

The function under the right part of the integral is the derivative of the function $\mathbf{F}(\zeta) / \mathbf{2}$ where $\mathbf{F}$ is given by:

$\mathbf{F}=-2 \Phi \sin (\theta)\left(q_{1} \cdot \cos (\theta) \cdot d-1\right) \cdot d+\left(q_{1} \zeta^{2} / 2+q_{1} \cdot d^{2} / 2\right.$

$\left.-q_{1} \cdot d^{2} \cos ^{2}(\theta)+\zeta+\cos (\theta) \cdot d\right) \ln \left(\zeta^{2}+2 \cos (\theta) \cdot d \cdot \zeta+d^{2}\right)$

$-q_{1} \cdot \zeta^{2} / 2+q_{1} \cdot d \cdot \zeta \cos (\theta)-2 \zeta$,

where $N_{q}=1 / 2\left(1+q_{1} \zeta\right)$ and the angle $\Phi$ is given by:

$\tan (\Phi)=\frac{\zeta+\cos (\theta) \cdot d}{\sin (\theta) \cdot d}$

$\left(\begin{array}{cc}M_{1}\left(e_{m}, e_{r}, 1,1\right) & \frac{1}{2}\left(M_{1}\left(e_{m}, e_{r}, 1,2\right)+M_{1}\left(e_{m}, e_{r}, 2,1\right)\right) \\ \frac{1}{2}\left(M_{1}\left(e_{m}, e_{r}, 1,2\right)+M_{1}\left(e_{m}, e_{r}, 2,1\right)\right) & M_{1}\left(e_{m}, e_{r}, 2,2\right)\end{array}\right)$.

(B) For the current matrix elements the contributions are:

$$
\begin{aligned}
M_{a b} & =M_{2}\left(e_{m}, e_{n}, e_{r}, p, q\right) \\
& =\int_{e_{m}} N_{p}(\xi) J(\xi) \mathrm{d} \xi\left\{\int _ { e _ { n } } H ( \mathbf { x } ( \xi ) , \mathbf { y } ( \eta ) ) \left[\int_{e_{r}}(G(\mathbf{z}(\zeta), \mathbf{y}(\eta))\right.\right. \\
& \left.\left.-G(\mathbf{z}(\zeta, \mathbf{x}(\xi))) N_{q}(\zeta) J(\zeta) \mathrm{d} \zeta\right] J(\eta) \mathrm{d} \eta\right\},
\end{aligned}
$$

with $a=m+p-1$ and $b=r+q-1$.

The contribution of each triplet of elements $e_{m}, e_{n}, e_{r}$ is built as previously by replacing $M_{1}\left(e_{m}, e_{r}, p, q\right)$ by $M_{2}\left(e_{m}, e_{n}, e_{r}, p, q\right)$.

The integrals $M_{2}$ contain a term where $H$ can be singular when $m=n, m=n+1$, or $m=n-1$. In fact, due to the regularization process, this singularity is removed by the term $(G(\mathbf{z}(\zeta), \mathbf{y}(\eta)-$ $G(\mathbf{z}(\zeta), \mathbf{x}(\xi)))$ which tends to zero when $\mathbf{y}(\eta)=\mathbf{x}(\xi)$. The problem is just that the numerical computation of the related terms contains undetermined terms. The cases $m=n+1$ or $m=n-1$ is related to a localized undetermined term at the contact between the elements $e_{m}$ and $e_{n}$, which is not a Gauss point and which therefore does not affect the numerical integration. The case $e_{m}=e_{r}$ can be treated numerically as explained in (Bonnet, 1995). The integration of $G$ on $e_{r}$ is obtained in a closed form:

Let us consider the interaction between point $\mathrm{O}(\mathbf{y}(\eta))$ and the segment $A B\left(e_{r}\right)$.

The integral of $G$ on $e_{r}$ can be written as:

$\left.\int_{e_{r}} \frac{-1}{4 \pi} \ln \left(\left\|\boldsymbol{V}+\mathbf{x}_{c}\right\|^{2}\right) N_{q}(\zeta) J(\zeta) \mathrm{d} \zeta\right]$

where $\mathbf{V}=\overrightarrow{A B} / 2$ and $\mathbf{x}_{c}$ is the relative position between $\mathbf{y}(\eta)$ and the center of $A B$ let us consider the integral $J$ defined by:

$J=\int_{e_{r}} \ln \left(\left\|\zeta . V+\mathbf{x}_{c}\right\|^{2}\right) N_{q}(\zeta) \mathrm{d} \zeta$. from this last result, the right integral is equal to $\frac{1}{2}(F(\zeta=1)-$ $F(\zeta=-1))$.

4.1.2. Computation of the potential function from the value of the harmonic function at boundary nodes

The nodal boundary values of $u$ may be obtained from the discrete form of (1) by:

$[u]=[G][\Phi]$,

where $[G]$ is the (regular) interaction matrix computed by discretization of (1).

$W=\frac{1}{2}[u]^{T}[K][u]$,

where the stiffness matrix $[K]$ is given by:

$[K]=\left[G^{-1}\right]^{T}[M]\left[G^{-1}\right]$.

This last relation allows the obtaining of the stiffness matrix as soon as the matrix $G$ has an inverse. It is known that, for plane problems, the existence of such an inverse needs some attention, which may lead in some cases to a scaling of the problem (see e.g. [9]) Under that form, the boundary harmonic function is obtained at collocation points, but its variation along the boundary is not known, because it is the repartition of the sources which is linear along each element. If the formulation is aimed at the construction of a boundary condition outside a finite element network, it does not seem that the boundary function $u$ thus obtained is the best solution. It seems better to look for an interpolation of the boundary potential which represents at the best the boundary potential induced by the sources and which is the best compatible with the interpolation used to compute the potential within the finite element mesh (linear for example). 

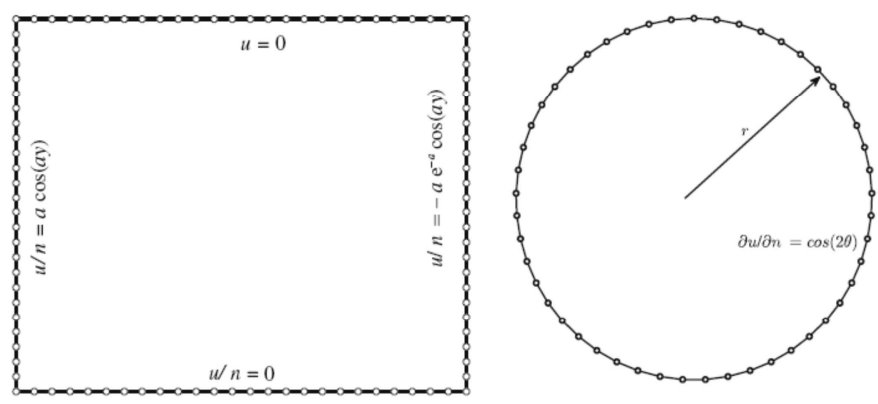

Fig. 1. Problem definition and BE discretizations for the two examples.

From Eq. (1), the value of the boundary potential can be obtained at any point along the boundary, for a given discretization of $\Phi$. Using the discretization (23), Eq. (1) becomes:

$$
u(\mathbf{y})=[M(\mathbf{y})][\Phi],
$$
(23)

where the matrix $[M(\mathbf{y})]$ is obtained by combination of (1) and

The nodal values of the linearized boundary potential are the values forming the matrix $[U]$ which contains the nodal values of the potential. For a given set of nodal values of the potential the approximate boundary linearized field is given by:

$$
\tilde{u}_{i}(\mathbf{y})=[N(\mathbf{y})][U] \text {. }
$$

The best choice of $[U]$ in $L^{2}$ is such that the integral $I_{1}$ is minimal, where

$I_{1}=\int_{\partial D}(\tilde{u}(\mathbf{y})-u(\mathbf{y}))^{2} \mathrm{~d} S(\mathbf{y})$

It leads to:

$[P][U]=[Q][\Phi]$,

where $[P]$ and $[Q]$ are given by:

$$
P_{i j}=\int_{\partial D} N_{i} N_{j} \mathrm{~d} S
$$

and

$$
Q_{i j}=\int_{\partial D} N_{i} M_{j} \mathrm{~d} S
$$

The matrix $P$ is easy to construct.

The different matrix elements of $Q$ are given by:

$$
Q_{i j}=\int_{e_{m}} \int_{e_{r}} G(\mathbf{z}(\zeta), \mathbf{x}(\xi)) N_{i}(\xi) N_{j}(\zeta) \mathrm{d} S(\zeta) \cdot \mathrm{d} S(\xi) .
$$

These terms are equal to the free terms of the matrix $M_{i j}$ given by Eq. (30).

\subsection{Case of the interior problem}

In the case of the interior problem, the formulation is exactly the same, just dropping the "free terms" obtained from (27) and changing the sign of the normal.

\section{Numerical examples}

Two numerical test examples are treated here in order to illustrate the effectiveness and the accuracy of the present symmetric boundary integral formulation. In the first one, the formulation is applied to an internal problem defined on a square domain while the second one concentrates on an external problem defined by an imposed flux over a circle. Fig. 1 shows the definition of the problems and their boundary element models.

\subsection{Example 1}

The main objective of this work is to build the stiffness matrix of a given domain, which is related, as explained before, to Dirichlet conditions. However, having built the stiffness matrix allows to deal with any kind of boundary condition and to check the ability of the stiffness matrix to produce a convenient relation between nodal potentials and fluxes. The function $u$ considered in this example is defined on the square domain $D=[0,1] \times[0,1]$ with the following boundary conditions:

$\frac{\partial u}{\partial \mathbf{n}}=\left\{\begin{array}{lll}a \cos (a y) & \text { on left edge } & x=0, \\ -a e^{-a} \cos (a y) & \text { on right edge } & x=1, \\ 0 & \text { on bottom edge } & y=0 .\end{array}\right.$

The constant $a$ is taken equal to $3 \pi / 2$ so that the potential function is null on the remaining top edge of the domain $(u(x, y=1)=$ 0 ). The boundary conditions related to given variations of $q(\mathbf{y})$ induce values of nodal fluxes whose matrix $[F]$ is given by a condensation on nodes using interpolation functions as:

$$
[F]=\int_{\partial D} q(\mathbf{y})[N(\mathbf{y})] \mathrm{d} S(\mathbf{y}) .
$$

It must be noticed that this approximation is perfectly compatible with the nodal condensation used in the FEM.

Dirichlet boundary conditions are taken into account by canceling the nodal values of $u$ at points located on the upper part.

The exact solution of this problem is $u=e^{-a x} \cos (a y)$.

A numerical boundary element model is built by dividing the squared boundary into linear elements of 0.04 element-length witch gives 25 elements per edge and a total of 100 elements for the whole closed boundary. The resulting assembled matrix $\mathbf{K}$ is reduced to a $74 \times 74$ square matrix after applying the boundary condition $u(x, y=1)=0$ and the right hand side vector $\mathbf{F}$ is assembled in the same way as it is done for the usual finite element assembly technique.

The approximated numerical solution on the left and bottom edges is shown in Fig. 2 with the corresponding exact curves, showing the good agreement between numerical and theoretical solutions.

\subsection{Example 2}

The problem in this second example concerns the determination of the potential field in a domain exterior to a circle of radius $R=1$ submitted to an imposed flux. 

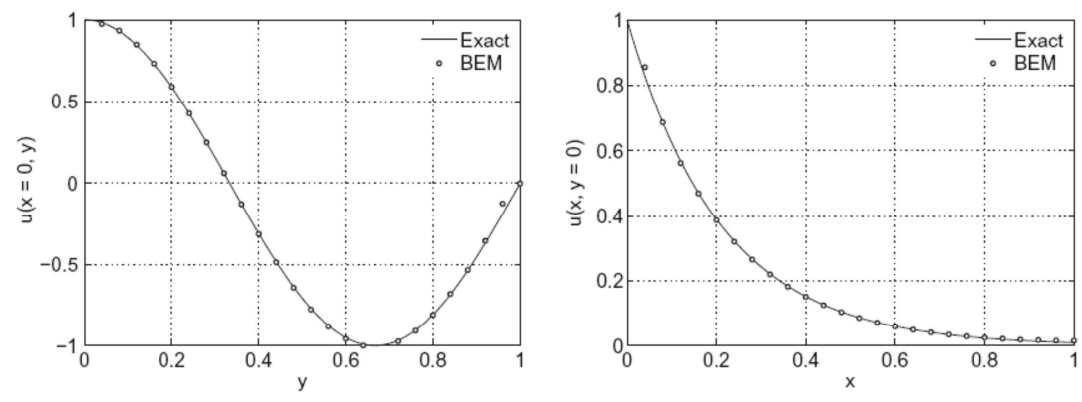

Fig. 2. $u(x, y)$ on the bottom edge and the left edge.

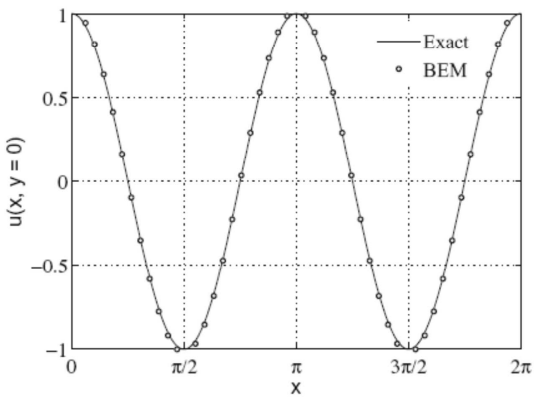

Fig. 3. Comparison of the numerical and exact solutions for the entire circle model.

$q(\theta)=\frac{\partial u(r=1, \theta)}{\partial \mathbf{n}}=\cos (2 \theta)$,

where the angle $\theta=\arctan (y / x)$ is measured from the $x$-axis and $r=\sqrt{x^{2}+y^{2}}$. The exact solution is $u=\cos (2 \theta) / r^{2}$.

The aim of this example is to test the free term in Eq. (16)

$\int_{\partial D} \Phi(\mathbf{x}) u(x) \mathrm{d} S(\mathbf{x})$,

which corresponds to the sum of single integrals over elements denoted by $e_{m}$ in Eq. (27):

\section{$\sum_{m} \int_{e_{m}} \Phi(\mathbf{x}(\xi)) u(\mathbf{x}(\xi)) \mathrm{d} \mathbf{x}(\xi)$}

These free terms produce the matrix $M_{\text {free. }}$. The stiffness matrix related to the external problem is then given by $M=M_{\text {int }}+M_{\text {free, }}$, where $M_{\text {int }}$ is the stiffness matrix for the interior problem.

The theoretical and computed solutions are shown in Fig. 3 displaying again a good agreement.

\section{Conclusion}

The method presented in this paper made it possible to build directly the boundary stiffness matrix related to a given domain for the Laplace operator. This result was obtained by expressing the potential function produced when a given boundary condition is applied at the boundary (Dirichlet boundary condition) and using a suitable repartition of sources at the boundary. This method is intermediate between the SGBEM method and the DtN FEM method. The first advantage of the method is to produce a symmetric stiffness matrix by avoiding the need to use as intermediate variables the boundary nodal fluxes at the boundary, which are not needed when the boundary stiffness matrix is required: the boundary stiffness matrix is indeed directly related to the potential function related to given boundary values of the potential. The second advantage is to produce a natural regularization method by using the classical regularization process introduced for the collocation method by [26] and to avoid the hypersingular kernels appearing in the SGBEM. In addition, the number of matrices to build in view to produce the final stiffness matrix is reduced. An example of application of the method in the case of Laplace equation within a plane domain has been provided, showing that the method leads to a convenient numerical solution of boundary value problems.

\section{References}

[1] F. Aliabadi, The boundary element method: Application in solids and structures, John Wiley and Sons, New York, 2002 .

B. Aour, O. Rahmani, M. Nait-Abdelaziz, A coupled FEM/BM approach and its accuracy for solving crack problems in fracture mechanics, Int J. Solids $S$ truct 4 (2007) $2523-2539$.

[3] M. Bonnet, M. Giuggiani, Direct evaluation of double singular integrals and new free terms in 2D (Symmetric) Galerkin BEM, Comput. Methods Appl. Mech. Engrg. 192 (2003) 2565-2596.

[4] M. Bonnet, G. Maier, C. Polizzotto, Symmetric Galerkin boundary element methods, Appl. Mech. Rev. 51 (11) (1998) 669-704.

[5] M. Bonnet, Regularized direct and indirect symmetric variational BIE formulations for three-dimensional elasticity. Engrg. Anal. Bound. Elem. 15 (1995) 93-102.

[6] M. Bonnet, Boundary Integral Equation Methods for Solids and Fluids, Wiley Publication, New York, 1999.

7] C.A. Brebbia, P. Georgiou, Combination of boundary and finite elements in elastostatics, Appl. Math. Modell. 3 (1979) 212-220.

[8] A. Carini, M. Diligenti, P. Maranezi, M. Zanella, Analytical integrations for twodimensional elastic analysis by the symmetric Galerkin boundary element method, Comput. Mech. 23 (1999) 308-323.

[9] S. Christiansen, On two methods for elimination of non-unique solutions of an integral equation with a logarithmic kernel, Appl. Anal. 13 (1982) 1-18

[10] T.G.B. Defigueiredo, A New Boundary Element Formulation in Engineering, Springer, Berlin, 1991

[11] F.M.E. Duddeck, M. Geisenhofer, A generalization of BEM by Fourier, Comput. Mech. 28 (2002) 303-310.

12] L. Gaul, F. Moser, Non-singular symmetric boundary element formulation for elastodynamics, Engrg. Anal. Bound. Elem. 25 (2001) 843-849.

[13] D. Givoli, J.B. Keller, A finite element method for large domains, Comput

[14] D. Givoli, Recent advances in the DtN FE method, Arch. Comput. Methods Engrg. 6 (2) (1999) 71-116.

[15] L. Halpern, O. Lafitte, Dirichlet to Neumann map for domains with corners and approximate boundary conditions, J. Comput. Appl. Math. 204 (2007) 505-

[16] Z.D. Han, S.N. Atluri, SGBEM-FEM alternating method for analyzing 3D surface cracks and their fatigue growth. Comput. Model. Engrg. Sci. 3 (6) (2002) 699-

[17] F. Hartmann, C. Katz, B. Protopsaltis, Boundary elements and symmetry, ngenieur Arch. 55 (1985) 440-449.

[18] T.J.R. Hughes, Multiscale phenomena: Green's functions, the Dirichlet-toNeumann formulation, subgrid scale models, bubbles and the origins of stabilized methods, Comput. Methods Appl. Mech. Engrg. 127 (1995) 387-401.

[19] D. Koyama, Error estimates of the DtN finite element method for the exterior Helmholtz problem, J. Comput. Appl. Math. $200(21-31)(2007) 321-331$. radiation and scattering of elastic waves in three dimensions, Int. J. Numer. Methods Engrg. 21 (1985) 115-129.

0] K.L. Leung, P.B. Zavareh, D.E. Beskos, 2D elastostatic analysis by a symmetric BEM/FEM scheme, Engrg. Anal. Bound. Elem. 15 (1995) 67-78.

[21] H.A. Mang, P. Torzicky, Z.Y. Chen, On the mechanical inconsistency of symmetrization of unsymmetric coupling matrices for BE-FEM discretizations of solids, Comput. Mech. 4 (1989) 301-308.

[22] T. Panzeca, F. Cucco, M. Terravecchia, Symmetric boundary element method versus finite element method, Comput. Methods Appl. Mech. Engrg. 191 (2002) 3347-3367.

[23] T. Panzeca, H. Fujita Yashima, M. Salerno, Direct stiffness matrices of BEs in the Galerkin BEM formulation, Euro. J. Mech. A/Solids 20 (2001) 277-298.

[24] C. Polizotto, M. Zito, Variational formulations for coupled BE/FE methods in elastostatics, Zeitsch. Angew. Math. Mech. 74 (1994) 533-543.

[25] C. Polizotto, An energy approach to the boundary element method, Comput. Methods Appl. Mech. Engrg. 69s (1988) 167-184.
S. Sirtori. G. Maier. G. Novati, S. Miccoli. Computational anisotropic elasticity. Int. J. Numer. Methods Engrg. 35 (1992) 255-282. boundary elements, Mechanica 14 (1979) 210-218.

[29] R. Springetti, G. Novati, M. Margonari, Weak coupling of the symmetric Galerkin BEM with FEM for potential and elastostatic problems, Comput. Model. Engrg. Sci. 13 (1) (2006) 67-80

[30] S. Terravecchia, Closed form coefficients in the symmetric boundary element approach, Engrg. Anal. Bound. Elem. 30 (2006) 479-488.

[31] J.L. Wearing, M.A. Sheikh, A regular indirect boundary element method for thermal analysis, Int. J. Numer. Methods Engrg. 25 (1988) 495-515.
[28] S. Sirtori, General stress analysis method by means of integral equations and 